\title{
¿Qué es el Control Predictivo y Hacia Dónde SE Proyecta?
}

\section{What is the Predictive Control and to Where it is Planning?}

\section{Sendoya Diego Fernando}

Escuela de Ciencias Básicas, Tecnología e Ingeniería, Universidad Nacional Abierta y a Distancia, Neiva, Colombia,

diego.sendoyalunad.edu.co.

\section{RESUMEN}

El control predictivo basado en modelo (Model Based Predictive Control - MBPC o simplemente $M P C$ ) es una metodología de control que hace uso del modelo del proceso para predecir las salidas futuras de la planta y con base en ello optimizar las acciones de control futuras. De hecho, el control predictivo no se puede considerar como una estrategia de control independiente sino, que por el contrario, integra toda una familia de métodos de control tales como, el control óptimo, el control de procesos con tiempos muertos, el control de procesos multivariables, etc. Esto ha permitido que el control predictivo haya tenido un desarrollo importante tanto en la comunidad científica y académica, como en el sector industrial.

Palabras clave: horizonte de control, horizonte de predicción, horizonte deslizante, modelo del proceso, respuesta base, respuesta óptima, trayectoria de referencia

\section{Abstract}

Model Based Predictive Control (MBPC or simply MPC) is a control methodology that uses the process model to predict the future plant outputs and optimize the future control actions. In fact, the predictive control cannot be considered as a separate control strategy but rather a family of integrated control methods such as optimal control, control of processes with dead time, multivariable processes control, etc. This has allowed predictive control has been an important development in both the scientific and academic community and in the industry.

Keywords: control horizon, prediction horizon, receding horizon, process model, base response, optimizing response, reference trajectory 


\section{INTRODUCCIÓN}

Hace algunas décadas, el único objetivo realmente importante en el control de procesos era lograr alcanzar una operación estable de la planta; sin embargo, hoy en día, la existencia de un mercado, tan variable y difícil de predecir, ha obligado a las empresas a mejorar sus procesos productivos conforme evoluciona el mercado, con el fin de mantenerse competitivas y rentables.

La competencia que existe en los diversos niveles de la industria, al igual que el creciente interés de la sociedad por los problemas del medio ambiente que se originan a raíz de los procesos industriales, han llevado a la necesidad de disponer de técnicas fiables que permitan la operación de la planta con alto grado de eficiencia y flexibilidad.

Actualmente, los sistemas de control industriales deben cumplir simultáneamente con los criterios de calidad en la producción, para satisfacer ciertas especificaciones sujetas a una demanda normalmente variable; con los criterios económicos, asociados con el mantenimiento de las variables de la planta y la minimización de una función de costos de operación; con los criterios de seguridad y con los criterios medioambientales.

Por lo tanto, en la actualidad, el objetivo de un sistema de control consiste en actuar sobre las variables manipuladas, de tal forma que puedan satisfacerse múltiples y cambiantes criterios de funcionamiento, en presencia de cambios en las características del proceso. Las diversas metodologías de control que existen hoy en día, se enfrentan al cumplimiento de este objetivo.

El control predictivo basado en modelo (MBPC - MPC) constituye una poderosa herramienta para afrontar este reto, ya que acepta cualquier tipo de modelo, funciones de costo o restricciones, convirtiéndose en la metodología que puede reflejar más directamente los múltiples criterios de funcionamiento, relevantes en la industria de procesos.
El control predictivo es un conjunto de métodos de control que se ha desarrollado alrededor de ciertos principios básicos comunes, tales como:

- El uso explícito de un modelo del proceso para pronosticar la salida del sistema en instantes futuros de tiempo.

- El cálculo de una acción de control óptima basado en la minimización de una o más funciones de costo, con la posibilidad de incluir restricciones sobre las variables del proceso.

Por lo tanto, los diferentes algoritmos miembros de la extensa familia MPC, difieren entre sí principalmente en:

- El tipo de modelo utilizado para representar el proceso y sus perturbaciones.

- La función de costo que ha de ser minimizada, con o sin restricciones.

Existen en la literatura varias anotaciones acerca del control predictivo aplicado a diversos procesos, como por ejemplo: generadores de vapor [1], aplicaciones en la industria de cemento [2], columnas de destilación [3], anestesia clínica [4] y robots [5].

El control predictivo presenta una serie de ventajas sobre otros métodos, entre las que se pueden citar las siguientes:

- Los principios de funcionamiento son intuitivos y los parámetros de diseño están orientados al desempeño, permitiendo que los conceptos puedan ser asimilados en un corto tiempo por los operarios de la planta, aún cuando se tengan pocos conocimientos en el área de control.

- Se pueden manejar problemas de control multivariable y no lineal de una manera sencilla.

- Actualmente, es la aproximación más natural al control restringido, un tema que cada día adquiere más importancia en el sector industrial 
(restricción de los actuadores, restricciones de seguridad, restricciones de calidad).

- Permite controlar procesos con comportamientos dinámicos poco usuales, tales como procesos de fase no-mínima, procesos altamente oscilatorios o procesos inestables.

- Su carácter predictivo lo hace compensar intrínsecamente los tiempos muertos.

- Introduce un control anticipativo, y de forma natural se compensan las perturbaciones medibles.

- Aunque se basa en ciertos principios básicos, es una metodología abierta que permite extensiones, contribuciones, alternativas y mejoras para el futuro.

Sin embargo, como es de esperarse, también presenta algunas desventajas:

- Pese a que su implementación no es compleja, resulta más difícil que la de los clásicos controladores PID.

- Si la dinámica del proceso es variable, la exigencia computacional para realizar los cálculos se incrementa; no obstante, con la potencia de los computadores actuales esta no es realmente una dificultad infranqueable.

\section{PrinciPIO de FUNCIONAMIENTO}

A continuación se describe la notación matemática que será utilizada a lo largo de la explicación:

- $t$ : representa el índice del tiempo discreto $(t=0,1,2, \ldots)$.

- $u(t)$ : representa la entrada del proceso (Variable manipulada).

- $y(t)$ : representa la salida del proceso (Variable controlada).
- $w(t)$ : representa el punto de operación (Setpoint).

- $u(t+k \mid t)$ : representa los valores futuros de la entrada, calculados en el tiempo $t$.

- $y(t+k \mid t)$ : representa los valores fututos de la salida con base en:

- Mediciones disponibles en el instante

$$
t:\{y(t), y(t-1), \ldots, u(t-1), u(t-2), \ldots\}
$$

- Valores futuros de la entrada en el instante

$$
t:\{u(t), u(t+1 \mid t), \ldots\}
$$

En la Fig. 1 se muestra el principio básico de funcionamiento del control predictivo, el cual se caracteriza por la siguiente estrategia:

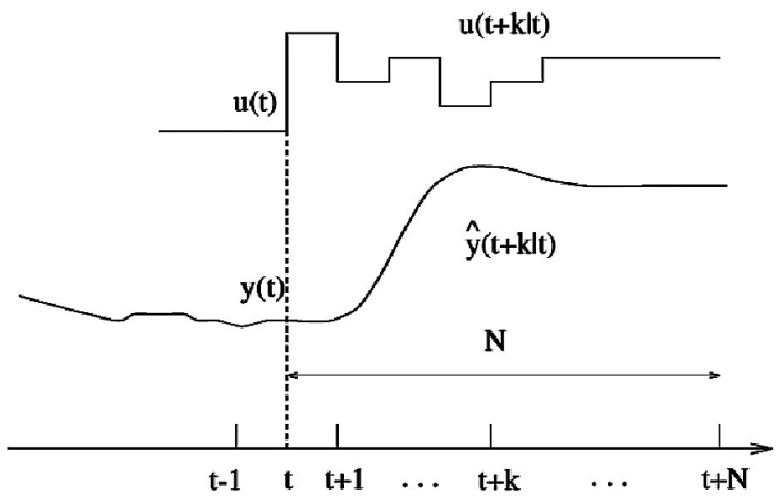

Fig. 1 Estrategia del MPC

1. A cada instante de tiempo "actual" $t$, se predice la salida del proceso $y(t+k)$ sobre un horizonte de tiempo $k=1 . . . N$.

$y(t+k \mid t)$ indica los valores futuros y el valor $N$ se conoce como el horizonte de predicción. La predicción se hace por medio del modelo del proceso, por lo que este debe estar disponible. Dicha predicción depende de las entradas y salidas pasadas, pero también del escenario de control futuro $\{u(t+k \mid t), k=0, \ldots, N-1\}$; es decir, las acciones de control que se aplicarán desde el momento presente $t$ en adelante. 
2. Se define una trayectoria de referencia $\{r(t+k \mid t), k=1, \ldots, N\}$, sobre el horizonte de predicción, que comienza en $r(t \mid t)=y(t)$ y evoluciona hacia el punto de operación $w$. Esta describe cómo se quiere guiar la salida del proceso desde su valor actual $y(t)$ hasta el punto de operación $w$. En caso de que el proceso presente tiempo muerto, es razonable iniciar dicha trayectoria después de este.

3. Se calcula el vector de control $\{u(t+k \mid t), k=0, \ldots, N-1\}$ para minimizar una función de costo específica, dependiendo de los errores de control predichos $\{[r(t+k \mid t)-y(t+k \mid t)], k=1, \ldots, N\}$.

4. El primer elemento $u(t \mid t)$ del vector de control óptimo $\{u(t+k \mid t), k=0, \ldots, N-1\}$ se aplica al proceso real en el instante de tiempo actual. Los demás elementos del vector de control calculado pueden ser despreciados $\mathrm{u}$ olvidados ya que en el siguiente instante de muestreo todas las secuencias de tiempo se desplazan y se obtiene una nueva medición de la salida $y(t+1)$ repitiendo todo el procedimiento nuevamente. Esto lleva a una nueva entrada de control $u(t+1 \mid t+1)$, la cual es generalmente diferente de la calculada previamente $u(t+1 \mid t)$; este concepto se conoce como horizonte deslizante.

En la Fig. 2 se presenta la estructura necesaria para implementar el control predictivo. Allí se usa un modelo de la planta, con el fin de predecir la evolución de la salida del proceso a partir de las señales de entrada y salidas conocidas. Las acciones de control futuras se calculan con el optimizador, que considera la función del costo y las posibles restricciones.

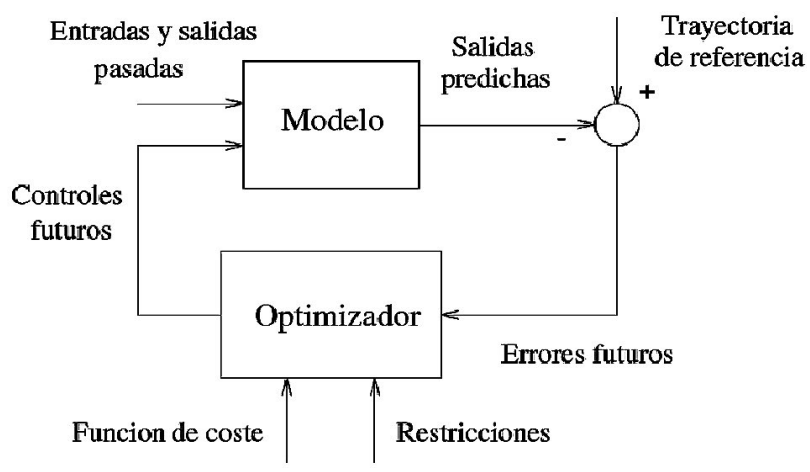

Fig. 2 Estructura básica del MPC

Como se puede apreciar, el modelo de proceso juega un papel decisivo en el controlador. Este debe ser lo suficientemente elaborado para capturar de la mejor manera posible la dinámica del proceso y, así, predecir de forma precisa la evolución del sistema; pero al mismo tiempo, debe ser lo suficientemente simple de implementar y entender, con el fin de reducir la carga computacional asociada al proceso de predicción. Las distintas metodologías de control predictivo difieren fundamentalmente en el tipo de modelo utilizado.

Otra parte fundamental de la estructura es el optimizador, el cual permite obtener las acciones de control por aplicar. Si el modelo es lineal, la función de costo es cuadrática y no se tienen restricciones; entonces se puede obtener una solución rápidamente y de manera precisa. Si por otra parte, este no es el caso, se ha de acudir al uso de algoritmos numéricos de optimización, lo cual incrementa la carga computacional al requerir mayor capacidad de cálculo.

Aunque el tamaño del problema resultante depende del número de variables del proceso, de los horizontes de control y predicción elegidos durante el diseño, y del número de restricciones que se consideran, en general, los problemas de optimización resultantes son problemas más bien modestos. 
Finalmente, se puede realizar una analogía entre la estrategia de control predictivo y la estrategia que se utiliza cuando se conduce un automóvil: el conductor conoce la trayectoria de referencia deseada para un horizonte de control finito. Tomando en consideración las características del automóvil (modelo mental del automóvil) decide qué acción de control tomar (acelerador, frenos, volante, marchas) para seguir la trayectoria deseada. Sólo la primera acción de control de la secuencia calculada mentalmente es aplicada por el conductor en cada instante y el procedimiento se repite en los sucesivos instantes utilizando el concepto de horizonte deslizante.

Por el contrario, cuando se utiliza un esquema de control clásico como PID, se utilizan sólo las señales pasadas. Esta forma de conducir el automóvil sería como conducir utilizando el espejo retrovisor, tal como se muestra en la Fig. 3.

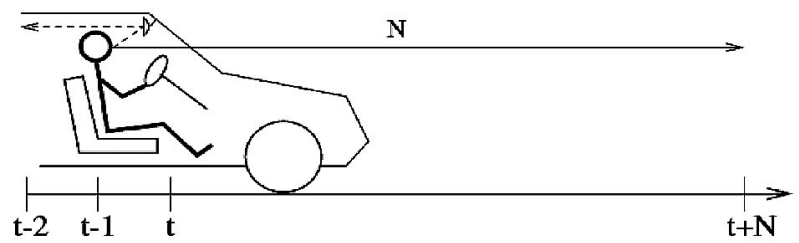

Fig. 3 Analogía $M B P C$

\section{Aplicaciones}

Algunas compañías utilizan controladores predictivos que han desarrollado para mejorar los procesos dentro de sus empresas y no los ofrecen externamente; sin embargo, existen otras que sí suministran dichos controladores. A continuación se destacan algunas, indicando el acrónimo del producto que proporcionan:

- AspenTech: Dynamic Matrix Control (DMC).

- Adersa:Identification and Command (ID-COM), Hierarchical Constraint Control (HIECON) y Predictive Functional Control (PFC).
- Honeywell Profimatics: Robust Model Predictive Control Technology (RMPCT) y Predictive Control Technology (PCT).

- Setpoint Inc.: Setpoint Multivariable Control Architecture (SMCA) y IDCOM-M (multivariable).

- Treiber Controls: Optimum Predictive Control (OPC)

- $\mathrm{ABB}: 3 \mathrm{dMPC}$.

- Pavillion Technologies Inc.: Process Perfecter

- Simulation Sciences: Connoisseur.

Hay que tener en cuenta que cada producto no es solamente un algoritmo, sino que viene acompañado por paquetes adicionales para la identificación de la planta o realización de pruebas.

Existen diversas aplicaciones del control predictivo en la industria. La mayor parte de las aplicaciones están en el sector petroquímico [6],[7] en los procesos de refinamiento, pero también existen numerosas aplicaciones en los sectores de pulpa y papel, procesado de alimentos, gas, minería, hornos, metalurgia, industria aeroespacial e industria automovilística. Una extensa recopilación teórica sobre control predictivo dirigida principalmente a personal de la industria con experiencia en control se puede encontrar en Rawlings [8].

Los resultados de un estudio realizado para la Society of Instrumentation and Control Engineering [9], son indicativos de las necesidades futuras de la industria en el ámbito del control. En este informe se analizan los principales problemas de control que se encuentran en la industria de procesos, el estado de aplicación de las tecnologías avanzadas, el grado de satisfacción de los usuarios con cada una de ellas y las expectativas que cada una genera. De acuerdo con el informe, 
los principales problemas que se presentan en el sector industrial tienen que ver con el retardo o tiempos muertos, las perturbaciones y la interacción entre variables. Del informe citado se puede extraer como conclusión interesante el hecho de que prácticamente todos los usuarios de control predictivo están satisfechos.

También resulta interesante intentar cuantificar la evolución futura de las distintas técnicas. En la Fig. 4 se intenta mostrar las posibilidades técnicas y las expectativas despertadas por cada una de ellas.

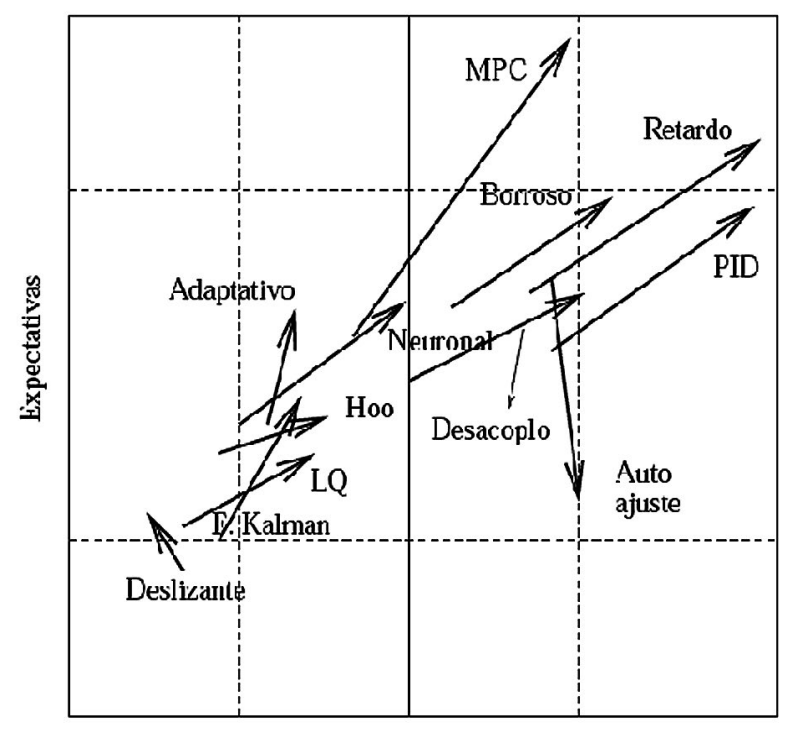

Fig. 4 Expectativas y posibilidades técnicas

Posibilidad técnica se refiere a la facilidad de implementación y expectativas, al efecto esperado en el uso de cada técnica. El citado artículo interpreta la flecha como tendencia futura. Según esto, el PID avanzado, compensación de retardo, borroso, desacoplo y MPC serán técnicas ampliamente usadas con grandes expectativas. EI control neuronal despierta grandes expectativas pero tiene ciertas dificultades de implementación, mientras que el Auto-ajuste se implementa con facilidad, pero pierde expectativas. Las técnicas como LQR, filtro de Kalman, $\mathrm{H}_{\infty}$ y Adaptativo se mantienen "sin demasiadas expectativas y no fácilmente implementables".

\section{CONCLUSIONES}

El control predictivo se considera una estrategia madura en el caso de procesos lineales, y se utiliza con bastante éxito en la industria. Sin embargo, no se puede considerar al control predictivo como una disciplina ya cerrada a la investigación, sino que, por el contrario, se trata de una rama de la ingeniería de control con gran auge hoy en día. Esto se puede constatar por el hecho de que en todos los congresos de control de estos últimos años siempre hay sesiones específicas de control predictivo y es muy poco el número de revistas de control en el que no aparezca algún artículo sobre este tema.

El control predictivo es todavía muy difícil de implementar en tiempo real para procesos no lineales, procesos híbridos o procesos muy rápidos, por lo que estos temas son objeto de investigación. En el caso de sistemas no lineales e híbridos han aparecido soluciones para cuestiones particulares y normalmente de pequeña dimensión, pero no existen soluciones generales. Sin embargo, si se trata de procesos lineales, el control predictivo puede ser aplicado inclusive cuando presenten dinámicas muy rápidas.

Un gran problema todavía no resuelto es el del análisis de estabilidad y robustez de los controladores predictivos; es decir, aun en el caso de que los controladores sean implementables, analizar cómo se puede asegurar su estabilidad en el caso nominal o en el caso de que el modelo no sea exacto. Además, en el caso de que el optimizador fuera capaz de encontrar una solución, no está garantizada la estabilidad del bucle cerrado.

Se han obtenido resultados que utilizan técnicas de control robusto en el contexto de controladores predictivos. La idea básica es tener en cuenta las incertidumbres sobre el proceso de una manera explícita y diseñar el controlador predictivo para optimizar la función objetivo ante la peor situación posible de las incertidumbres. 
En cualquier caso, estos resultados exigen el cómputo de regiones invariantes que salvo en el caso de sistemas lineales son difícilmente calculables.

Estos resultados prometedores permiten pensar que el control predictivo experimentaría una mayor diseminación tanto en el mundo académico como en la comunidad industrial en los próximos años.

\section{RefERencias}

[1] J. Richalet, A. Rault, J.L. Testud y J. Papon, "Model Predictive Heuristic Control: Application to Industrial Processes,", Automatica, vol. 14 (2), pp. 413-428, 1978.

[2] D.W. Clarke, "Application of Generalized Predictive Control to Industrial Processes," IEEE Control Systems Magazine, vol 122, pp. 49-55, 1988.

[3] J. Richalet, "Industrial Applications of Model Based Predictive Control,', Automatica, vol 29 (5), pp. 12511274, 1993.

[4] D.A. Linkers y M. Mahfonf, "Advances in Model-Based Predictive Control,' en Generalizad Predictive Control in Clinical Anaesthesia, Oxford University Press, 1994.

[5] J. Gómez Ortega y E.F. Camacho, "Mobile Robot Navigation in a Partially Structured Environment using Neural Predictive Control,', Control Engineering Practice, vol 4, pp. 1669-1679, 1996.

[6] S.J. Qin y T.A. Badgwell, "An Overview of Industrial Model Predictive Control Technology," en AIChE Symposium Series, pp. 232-256, 1997.

[7] S.J. Qin y T.A. Badgwell, "An Overview of Nonlinear Model Predictive Control Applications", en IFAC Workshop on Nonlinear Model Predictive Control. Assessment and Future Directions, Ascona, Suiza, 1998.

[8] J. Rawlings, "Tutorial Overview of Model Predictive Control,' IEEE Control Systems Magazine, pp. 38-52, 2000.

[9] H. Takatsu, T. Itoh y M. Araki, "Future needs for the control theory in industries. Report and topics of the control technology survey in japanese indsutry,", Journal of Process Control, vol 8, pp. 369-374, 1998. 